\title{
'Fish'ing for regulators of inflammation
}

Inflammation is an important part of pathogen clearance and tissue repair, but incomplete resolution of the inflammatory process damages the surrounding tissue and contributes to chronic diseases such as rheumatoid arthritis, atherosclerosis and chronic obstructive pulmonary disease. So, there has been considerable interest in strategies to enhance inflammatory resolution, which could reduce pathogenic chronic inflammation without affecting the immune system's ability to respond to new challenges. In a recent paper in Science Translational Medicine, a natural product known as tanshinone IIA was found to promote the resolution of inflammation by inducing neutrophil apoptosis and reverse migration.

To identify compounds that regulate inflammatory resolution, sterile tissue injury was used to produce an inflammatory response in zebrafish embryos that expressed green fluorescent protein (GFP) from a neutrophil-specific promoter. When the inflammatory response started to peak, the zebrafish were exposed to molecules from a compound library, and GFP-positive cells at the site of injury were counted during the resolution stage. Compounds with known anti-inflammatory activity, including inhibitors of phosphoinositide 3-kinase (PI3K) or p38 mitogenactivated protein kinase (p38 MAPK), reduced the number of GFP-positive cells at the wound, as did nine compounds that were not previously known to have a role in inflammation. Two of these - tanshinone IIA and cryptotanshinone - are structurally similar, and tanshinone IIA is found naturally in Salvia miltiorrhiza, a Chinese medicinal herb used to treat cardiovascular disease.

Once the pro-inflammatory signal subsides, neutrophils either apoptose or migrate away from the signal; tanshinone IIA increases both of these processes. In freshly isolated human neutrophils, tanshinone IIA induces apoptosis, even in the presence of neutrophil pro-survival stimuli such as granulocyte-macrophage colonystimulating factor (GM-CSF) and activators of hypoxia signalling.

In their zebrafish model, there was little evidence of apoptotic neutrophils, perhaps because they are rapidly phagocytosed by macrophages at the site of inflammation. The contribution of apoptosis to inflammation resolution in this model did not fully explain the observed changes in neutrophil number, so the authors investigated other possible mechanisms of resolution. Zebrafish neutrophils were engineered to express a photoactivatable fluorescent protein known as Kaede, which irreversibly changes in colour from green to red upon stimulation with ultraviolet light. Neutrophils that were photoconverted at the site of injury were subsequently found to leave the site of injury more frequently in zebrafish treated with tanshinone IIA than in those treated with vehicle. Thus, in the zebrafish sterile tissue injury model, much of the tanshinone IIA-induced resolution of inflammation was attributable to neutrophil reverse migration.

Chemorepulsion, increased cell mobility or reduced sensitivity to chemoattractive agents could all cause neutrophils to move away from the wound. The authors therefore tracked the migrating tanshinone IIA-treated neutrophils to distinguish between these modes of action. The neutrophil movements were similar to those predicted by mathematical models of diffusion (ruling out chemorepulsion) but they did not move any faster than untreated cells. The increased reverse migration observed in zebrafish treated with tanshinone IIA may therefore be attributable to an induced insensitivity to chemoattractive agents released from the wound. Notably, tanshinone IIA did not affect the ability of neutrophils to move towards the site of injury, which suggests that the response to de novo immunological challenges would be unaffected by this compound.

Overall, as well as finding that tanshinone IIA and related compounds can mediate inflammation resolution, this study demonstrates that zebrafish can be used as a relatively high-throughput in vivo screening platform for novel anti-inflammatory drugs. The molecular mechanism still needs to be identified, but if these effects are also observed in clinical trials then tanshinone IIA could become an important therapeutic treatment for diseases caused by incomplete resolution of inflammation.

Megan Cully

ORIGINAL RESEARCH PAPER Robertson, A. L.

et al. A zebrafish compound screen reveals modulation of neutrophil reverse migration as an anti-inflammatory mechanism. Sci. Transl. Med. 6 , 225ra29 (2014) 